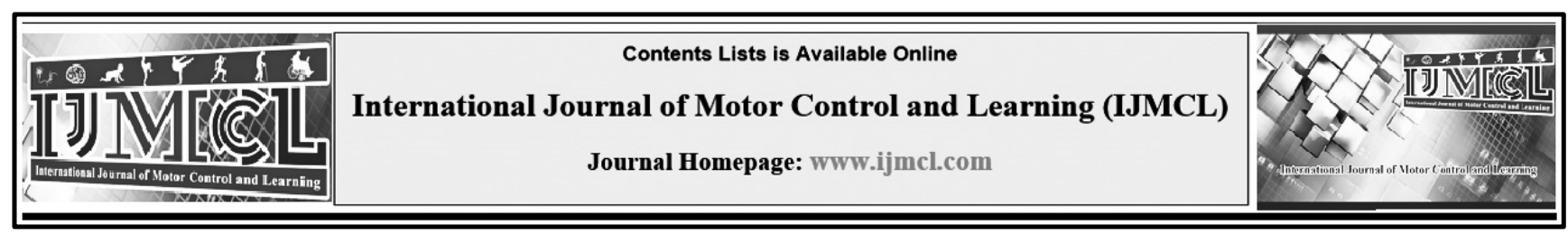

\title{
The Effect of Mental Fatigue on Three-Point Shot Performance in Skilled Basketball Players
}

\author{
Alireza Bahrami ${ }^{\mathrm{a}}$, Jalil Moradi ${ }^{\mathrm{a}}$, Zahra Etaati ${ }^{\mathrm{b}}$ \\ ${ }^{a}$ Department of Motor Behavior and Sport Psychology, Faculty of Sport Sciences, Arak University, Arak, Iran \\ ${ }^{\mathrm{b}}$ M.Sc., Sport Psychology, University of Tehran, Tehran, Iran
}

\begin{tabular}{l}
\hline Keywords \\
\hline Basketball \\
Performance \\
Three-Point Shoot \\
Mental Fatigue
\end{tabular}

\begin{abstract}
Background: Mental fatigue is a psychobiological state caused by prolonged periods of demanding cognitive activity.

Objective: This study aimed to investigate the effect of mental fatigue on three-point shots in skilled basketball players.

Methods: This study was semi-experimental. For this purpose, 18 male experienced basketball players were selected by convenience sampling and randomly divided into experimental and control groups (control: $n=9$, experimental: $n=9$ ). Participants in the experimental group experienced mental fatigue using strop software and math tests, but the control group did not perform any specific cognitive tasks during this time. Visual Analog Scale and three-point shots were used as a pretest and posttest. For data analyzing, the SPSS 25 was applied, and the significance level was considered at $(\mathrm{p}<0 / 05)$.

Results: The results of the study illustrated that spatial working memory played a predictive role in selected attention (same color and font, and different color and font) and performance accuracy; Moreover, mathematical working memory played a predictive role in selected attention (same color and font, and different color and font). However, it did not have a predictive role in performance accuracy.

Conclusion: The result of this study supports the theory that mental fatigue had impact on athletic performance. It is suggested that basketball players and coaches consider mental fatigue in sports performance.
\end{abstract}

Zahra Etaati,

Email: z.etaati92@gmail.com

Received: 2020/07/25

Accepted: 2020/10/09

Published: 2020/11/05

\section{Introduction}

Mental fatigue is a sense experienced and felt during or after long periods of cognitive activities and determined by tiredness and energy deficiency (Marcora et al., 2019; Zamani et al., 2017). Fatigue is classified into two types of physical and mental. Physical fatigue is a physical weakness caused by repetitive muscular activities. Mental fatigue, on the other hand, is seen as a decrease in efficiency in productivity of the mind (Chaudhuri \& Behnam, 2004). This acute fatigue is associated with longterm mental effort and differs from chronic fatigue and cognitive impairment related to aging or illness
(Ackerman et al., 2009; McMahon et al., 2014). The effects of mental fatigue can be divided into three distinct categories: mental, behavioral, and physiological responses. When tired, participants usually complain of less work capacity, unwillingness to try to do homework, reduced perceptions of personal performance, and mental distress and fatigue (Desmond \& Hancock, 2001). Studies have shown that the effects of mental fatigue are associated with reduced ability to process information, maintain attention, anticipate actions, and make decisions (Kunrath et al., 2018). 
Marcora et al. (2009) showed the first empirical evidence that fatigue caused by a mental effort can impair physical function. Mental fatigue is also associated with a wide range of "side effects": exhaustion, increased fat, feelings of compassion, decreased sense of motivation and alertness, and changes in perception and mood; Therefore, it has been suggested that the consequences of mental fatigue has two sides: it may prohibit performance by increasing the feeling of tiredness "I can't do this, I'm tired" or by devaluing the importance of success in a specific case: "I don't feel good about doing it, it's not worth it" (Dantzer et al., 2014). Studies in football have shown that mental fatigue primarily impairs physical and technical performance (Kunrath et al., 2018). Smith et al. (2015) performed a study on ten people (including footballers, Australian football players, and rugby players) was carried out in the form of a walking protocol with six different intensities (standing, strolling, running, running fast, and sprinting on a treadmill). In mental fatigue, comparing players' performance between control and experimental conditions, the results showed a shorter distance as well as a lower average speed in low-speed conditions (such as walking). In another study, Smith et al. (2016) examined the effect of mental fatigue on the maximum physical abilities of recreational soccer players. During mental fatigue, individuals showed a $16.3 \%$ decrease in intermittent $\mathrm{U}-\mathrm{U}$ test. In the case of technical variables, it was found that beyond physical dysfunction, mental fatigue also affected technical performance. In a technical test with regular kicks and passes, the players provided penalties with more defects as well as low accuracy kicks and decreased speed of the ball while scoring. Rahimizadeh et al. (2017), in a study, examined the effects of different levels of mental fatigue on the emergence of outstanding skills in free throws of basketball in male students. The study was conducted with the participation of university basketball players at three levels of mental fatigue (low, medium, and high). Subjects $(\mathrm{N}=8$ in each group) performed 120 throws from seven angles, with 20 throws from each angle at three levels of mental fatigue. Data analysis showed that a low mental fatigue group performed better than other groups. The study looked at the effect of mental fatigue on heart rate changes, measuring mental fatigue and swimming performance in young athletes. The results showed that the induction of mental fatigue reduces swimming performance by $1500 \mathrm{~m}$ and does not alter heart rate changes (Penna et al., 2018).

The results of Al-Rokan et al. (2017) study showed the negative effect of mental fatigue on specific skills such as free throwing in basketball. Another study looked at whether mental fatigue reduced free throw points in basketball. In this study, 18 semi-professional players took the test. The results showed that participants in the experimental group had a lower success rate than the control group (Lopez et al., 2017).

Various studies have examined the different effects of mental fatigue on athletes' performance. While a lot of them have shown that mental fatigue reduces athletes' performance, there are also some researches showing contradictory results. Smith et al. (2015), for example, conducted a study to 
investigate the effect of mental fatigue on intermittent running performance. The study found that mental fatigue did not significantly affect running speeds and peak speeds. In the study by Van Cutsem et al. (2017), it was stated that maximum strength, power, and anaerobic work are not affected by mental fatigue. In addition, in the research of Rahimizadeh et al. (2017), it has been determined that due to the emergence of outstanding skills, despite mental fatigue, specific parameters remain strong and lead to the emergence of exceptional skills.

Basketball faces many cognitive needs and creates an environment in which players are forced to perform continuous mental operations to respond to environmental demands. Hence, they need a lot of mental effort (Cardenas, 2015). In the case of the effect of mental fatigue on the skill of throwing a three-point shot at skilled athletes, limited study records. Also, due to the importance of mental fatigue, it is necessary to repeat and describe more studies in this field. Therefore, the present study seeks to investigate the effect of mental fatigue on the performance of three-point shooting skills in skilled basketball players.

\section{Method}

This research is semi-experimental and practical in terms of purpose. The research project was also in the form of pretest, posttest, and was conducted in two experimental and control groups. The population of the study consisted of all male basketball players, 18 of whom, with average and standard deviation of $(22.2 \pm 3.35)$, were selected based on entry-exit criteria and using convenient sampling from Aluminum Arak sports club, Arak, Iran. They were then randomly assigned to two groups, each containing nine persons. The inclusion criteria for the research were having at least three years of experience in the field of basketball under the supervision of a professional coach, healthy physical condition, and regular physical activity in the club. All athletes voluntarily participated in this study, and written consent was obtained before experiments. The criterion for leaving the research was the dissatisfaction of the subjects.

\section{Measurement Tool}

To create mental fatigue in athletes, complex strop software, as well as mathematical tests based on four primary operations and the use of power two, was used (Arqami et al., 2013). Visual Analog Scale (VAS) was used to assess mental fatigue (Arqami et al., 2013). On this scale, the number zero indicates the highest level of fatigue, and the number ten indicates the lowest level of mental fatigue (the highest level of consciousness). Performance evaluation was performed using the three-point shot. The ground was set for throwing the basketball and was determined by the degree with different angles at the three-point throw point relative to the ring.

\section{Implementation Method}

Athletes were first invited to attend a meeting to explain the goals and how to conduct research and gain satisfaction. Subsequently, they were randomly divided into two groups of nine. At the beginning of the research session, each of the 
subjects was given a preliminary assessment of mental fatigue using the VAS self-expression scale and the individual performance test. According to the purpose of the study, people in the experimental group should suffer from mental fatigue, and their mental fatigue scores should be higher than average. For this purpose, after the initial assessment of mental fatigue, participants in the experimental group were given strop software exercises as well as math tests. To achieve high mental fatigue, each of the participants performed these exercises for about 120 minutes (Rahimizadeh, Shahbazi, Tahmasebi Boroujeni, 2017). The subjects in the control group did not perform cognitive and physical activity during this period. Participants in both groups were then assessed for mental fatigue assessment (to determine the extent of mental fatigue) and performance test (individual test) as a posttest.
Pretests, mental fatigue tests, and posttests were performed on each of the subjects in one session.

\section{Statistical Methods}

Descriptive statistics (mean and standard deviation) were used to describe the variables of the study and to draw the table and graph diagram. The information obtained was analyzed using Ttests for correlated and independent groups after ensuring that the data were standard. In all statistical tests, the significance level was considered $\mathrm{P}<0.05$.

\section{Results}

Table 1 shows the mean and standard deviation of the mental fatigue scores and the performance of the participants in three-points shots in the pretest and posttest in both experimental and control groups.

Table 1. Mean of the groups in terms of the variables of mental fatigue and the performance of the three-point basketball shots during the test stage.

\begin{tabular}{cccccc}
\hline \multirow{2}{*}{ Groups } & Test Stages & \multicolumn{2}{c}{ Performance } & \multicolumn{2}{c}{ Mental Fatigue } \\
\cline { 3 - 5 } & Mean & $\begin{array}{c}\text { Standard } \\
\text { Deviation }\end{array}$ & Mean & Standard Deviation \\
\hline $\begin{array}{c}\text { Experimental } \\
\text { Group }\end{array}$ & Pretest & 34.18 & 3.79 & 8.44 & 1.33 \\
\cline { 2 - 5 } & Posttest & 29.70 & 4.49 & 3.67 & 0.5 \\
\hline $\begin{array}{c}\text { Control } \\
\text { Group }\end{array}$ & Pretest & 34.92 & 1.97 & 8.56 & 1.42 \\
\cline { 2 - 5 } & Posttest & 33.29 & 1.90 & 8.33 & 0.86 \\
\hline
\end{tabular}

Table 2. Average score of basketball shooting in pretest and posttest.

\begin{tabular}{cccccccc}
\hline \multirow{2}{*}{ Variables } & \multicolumn{2}{c}{ Pretest } & \multicolumn{2}{c}{ Posttest } & \multicolumn{3}{c}{ Paired t-test } \\
\cline { 2 - 7 } & Mean & $\begin{array}{c}\text { Standard } \\
\text { Deviation }\end{array}$ & Mean & $\begin{array}{c}\text { Standard } \\
\text { Deviation }\end{array}$ & $\begin{array}{c}\text { Level of } \\
\text { Freedom }\end{array}$ & $\begin{array}{c}\mathrm{T} \\
\text { Value }\end{array}$ & $\begin{array}{c}\text { Level of } \\
\text { Significance }\end{array}$ \\
\hline $\begin{array}{c}\text { Experimental } \\
\text { Group }\end{array}$ & 34.18 & 3.79 & 29.70 & 4.49 & 8 & 2.81 & 0.02 \\
\hline $\begin{array}{c}\text { Control } \\
\text { Group }\end{array}$ & 34.92 & 1.97 & 33.29 & 1.90 & 8 & 3.13 & 0.01 \\
\hline
\end{tabular}


Table 1 shows that in the three-point shots of basketball and mental fatigue in the experimental group, there was a significant decrease from pretest to posttest. But there is no noticeable difference in the control group. A reduction in mental fatigue scores means an increase in fatigue, which indicates that the rate of mental fatigue has increased after the application of the fatigue protocol. Before analyzing the data with statistical tests, the Shapiro Wilk test was used to examine the naturalness of the data, and the results showed that the data had a natural distribution. Then, to ensure that the athletes in the experimental group did get tired, the fatigue scores were compared before and after applying the fatigue protocol. The results showed that there was a significant difference between the mean scores of the experimental group (/df-(8_-9/16, P$0.001)$; however, there was not a significant difference in the control group $(8+-0 / 80$, P$0.44)$.

To analyze the data, first, the mean of the experimental and control groups in the threepoint basketball shot in the pretest was compared using the independent t-test, which showed that there is no significant difference in in the three-point basketball shot (/df-(16-0.51, $\mathrm{P}-0.61)$ and in mental fatigue (/df-(16-0.17, P0.86 ) between the two groups. To compare the groups in the pretest and posttest, the T-test for correlated groups was used in the three-point shot variable, the results of which are presented in Table 2. To compare the groups in the pretest and posttest stages, the T-test for the threepoint shot variable was used in the paired groups, the results of which are presented in Table 2.

The results of Table 2 show that there is a significant difference between the mean scores of the two groups in the pretest and posttest scores because the calculated $\mathrm{T}$ value is greater than the T-value with freedom of 8 and a probability level of 0.05 . Therefore, the existence of a statistically significant difference between the pretest and posttest scores in the three-point basketball shooting skill is accepted in both groups. But a comparison of the mean groups in the posttest with the independent $\mathrm{T}$ test showed that there was a significant difference between the experimental and control groups $(\mathrm{P}=0.04$, df $=(16)=-2.20)$. These results show that the reduction in the experimental group was significantly higher than the control group. In other words, mental fatigue has had a significant effect on reducing the performance of skilled basketball players.

\section{Discussion and Conclusion}

This study aimed to investigate the effect of mental fatigue on the performance of three-point shot skills in skilled basketball players. The results show a decrease in the quality of performance, and it was found that mental fatigue affected maintaining a high level of performance.

According to the results reported in Table 1, it seems that our mental fatigue manipulation has been successful. Participants in the experimental group felt mentally exhausted after taking the strop 
and math tests. As athletes' levels of fatigue increased, they became less interested in continuing to perform tasks with mental fatigue, which is an essential feature of mental fatigue. They were followed by a three-point shot performance test. In addition, each participant performed three repetitions of this test, while the participants were told that they could throw the ball with their preferred speed and accuracy. Participants did endurance training after mental fatigue. In a 2017 study by Van Cutsem, it was found that mental fatigue is more palpable in endurance sports. Therefore, the results of this study confirm the research of Van Cutsem (2017). Concerning the effect of mental fatigue on athletes' performance in three-point basketball shots, the present study is consistent with the research of Marcora and Kots (2015), Smith et al. (2016), (Penna et al., 2018), Al-Rokan et al. and Lopez et al. (2017).

The results of these studies have shown that mental fatigue has significant effects on athletes' technical and tactical performance. In other words, mental fatigue is associated with influencing variables such as the decreased ability to process information, attention retention, predictability, and decision making (Kunrath et al., 2018) and, according to Marcora et al. (2009), fatigue caused by a mental effort can impair physical function. As mentioned previously, mental fatigue is also associated with a wide range of "side effects": lack of energy, increased fat, and feelings of compassion, decreased sense of motivation and alertness, and changes in perception and mood;
Therefore, it has been suggested that the consequences of mental fatigue may be twofold (Dantzer, 2014). It is possible that the imposition of a cognitive burden on basketball players by using Strop software exercises and math tests has also affected their energy levels, and these effects have overshadowed physical performance and throwing the third shot. The results of this study are inconsistent with those of Smith et al. (2015) and Boroujeni et al. (2017). In this study, it was noted that the peak performance and performance of skilled athletes are not affected by mental fatigue. One of the limitations of the present study was the use of the VAS scale in mental 1 fatigue assessment, which is a self-assessment test, so the results are not very sensitive and do not specify the exact level of mental fatigue assessment, which is a self-assessment test, so the results are not very sensitive and do not specify the exact level of mental fatigue. Therefore, it is recommended to consider this point in future research and use other fatigue assessments in addition to the selfassessment scale. In this study, other groups, including amateur athletes, were not present to assess the effect of mental fatigue and to compare with the group of skilled athletes. Therefore, it is recommended that this issue be considered in future researches.

Overall, the results of this study confirm that mental fatigue affects athletic performance. Because mental fatigue hurts athletes' athletic performance; Athletes, coaches, and other sports managers should pay attention to this rationale and look for ways to prevent and improve mental fatigue during a sports activity that has a cognitive 
load. For example, to optimize performance, it is necessary to avoid doing mental work before competitions that require endurance (Van Cutsem, 2017).

\section{References}

1. Ackerman, P. L., \& Kanfer, R. (2009). Test length and cognitive fatigue: an empirical examination of effects on performance and test-taker reactions. Journal of Experimental Psychology: Applied, 15(2), 163.

2. Alarcón, F., Ureña, N., \& Cárdenas, D. (2017). La fatiga mental deteriora el rendimiento en el tiro libre en baloncesto. Revista de psicología del deporte, 26(1), 3336.

3. Arghami, S., Ghoreishi, A., Kamali, K., \& Farhadi, M. (2013). Investigating the consistency of mental fatigue measurements by visual analog scale (vas) and flicker fusion apparatus. Iranian Journal of Ergonomics, 1(1), 66-72. (in Persian).

4. Cárdenas, D., Conde-González, J., \& Perales, J. C. (2015). El papel de la carga mental en la planificación del entrenamiento deportivo. Revista de psicología del deporte, 24(1), 91-100.

5. Chaudhuri, A., \& Behan, P. O. (2004). Fatigue in neurological disorders. The lancet, 363(9413), 978-988.

6. Dantzer, R., Heijnen, C. J., Kavelaars, A., Laye, S., \& Capuron, L. (2014). The neuroimmune basis of fatigue. Trends in neurosciences, 37(1), 39-46.

7. Desmond, P. A., \& Hancock, P. A. (2001). Active and passive fatigue states.

8. Hopkins, D., Shick, J., \& Plack, J. (1984). Aahperd skills test manual: Basketball for boys and girls. Reston, VA: AAHPERD .

9. Kunrath, C. A., Cardoso, F., Nakamura, F. Y., \& Teoldo, I. (2018). Mental fatigue as a conditioner of the tactical and physical response in soccer players: A pilot study. Human Movement, 19(3), 16-22.

10. López, F. A., Ortín, N. U., \& Vélez, D. C. (2017). Mental fatigue impairs the basketball free-throw performance. Revista de Psicologia del Deporte, 26(3), 33-36.

11. MacMahon, C., Schücker, L., Hagemann, N., \& Strauss, B. (2014). Cognitive fatigue effects on physical performance during running. Journal of Sport and Exercise Psychology, 36(4), 375-381.

12. Marcora, S. M., Staiano, W., \& Manning, V. (2009). Mental fatigue impairs physical performance in humans. Journal of applied physiology, 106(3), 857-864.

13. Penna, E. M., Wanner, S. P., Campos, B. T., Quinan, G. R., Mendes, T. T., Smith, M. R., \& Prado, L. S. (2018). Mental fatigue impairs physical performance in young swimmers. Pediatric exercise science, 30(2), 208-215.

14. Rahimizadeh, M., Shahbazi, M., Tahmasebi Boroujeni, S. (2017). The effect of different levels of mental fatigue on the emergence of outstanding skills of free basketball throws for male students. Journal of Sports Psychology Studies, No. 22, pp. 14-1. (in Persian).
15. Smith, M. R., Coutts, A. J., Merlini, M., Deprez, D., Lenoir, M., \& Marcora, S. M. (2016). Mental fatigue impairs soccer- specific physical and technical performance. Medicine \& Science in Sports \& Exercise, 48(2), 267-276.

16. Smith, M. R., Marcora, S. M., \& Coutts, A. J. (2015). Mental fatigue impairs intermittent running performance. Medicine \& Science in Sports \& Exercise, 47(8), 1682-1690.

17. Van Cutsem, J., Marcora, S., De Pauw, K., Bailey, S., Meeusen, R., \& Roelands, B. (2017). The effects of mental fatigue on physical performance: a systematic review. Sports medicine, 47(8), 1569-1588.

18. Zamani G, Doostan M. (2017). The effect of Transcranial Direct Current Stimulation on Working Memory and Reactiontime in Athlete Girls. Neuropsychology, 10(3), 51-62. (in Persian). 